\title{
Lipoxygenase-Generated Icosanoids Inhibit Glucose-induced Insulin Release from Rat Islets
}

\author{
M. H. Nathan* and S. Belbez Pek ${ }^{\dagger}$ \\ ${ }^{*}$ Department of Internal Medicine (Division of Endocrinology and Metabolism), University of \\ Michigan, Ann Arbor, Michigan, USA and ${ }^{\$ 5560}$ Medical Sciences Research Building-2, University \\ of Michigan Medical Center, Ann Arbor, MI 48109-0678, USA (Correspondence to SBP)
}

$A B S T R A C T$. Lipoxygenase-pathway metabolites of arachidonic acid are produced in pancreatic islets. They are are implicated in insulin release, since nonselective inhibitors of lipoxygenases inhibit glucose-induced insulin release. We studied the interplay in insulin release between glucose and selected icosanoids formed in 5-, 12- and 15-lipoxygenase pathways. Effects on immunoreactive insulin release of $10^{7}$ to $10^{6}$ 12-(R)-HETE, 12-(S)-HETE, hepoxilin $\mathrm{A}_{3}$, lipoxin $\mathrm{B}_{4}, \mathrm{LTB}_{4}$ or $\mathrm{LTC}_{4}$ were tested individually in 30 -min incubations of freshly isolated young adult Wistar rat pancreatic islets, in the presence of $5.6 \mathrm{mM}$ or $23 \mathrm{mM}$ glucose. Basal insulin release (at $5.6 \mathrm{mM}$ glucose) was stimulated by $\mathrm{LTC}_{4}$ and hepoxilin $\mathrm{A}_{3}(304 \%$ and $234 \%$ of controls at $5.6 \mathrm{mM}$ glucose alone, respectively), inhibited by 12-(S)-HPETE (56\%), and was not affected by 12-(R)-HETE, 12-(S)-HETE, lipoxin $B_{4}$ or $L^{2} B_{4}(11 \%$, 105\%, $106 \%$ and $136 \%$, respectively). Insulin release evoked by $23 \mathrm{mM}$ glucose $(190-320 \%)$ was inhibited $(50-145 \%)$ by all icosanoids tested, except LTC $(162 \%)$. We conclude that, among the lipoxygenase products tested, only leukotrienes and hepoxilin are candidates for a tonic-stimulatory influence on basal insulin release. Since glucose promotes icosanoid formation in islets, the observed inhibition of glucose-induced insulin release by lipoxygenase products suggests the existence of a negative-feedback system.

\section{INTRODUCTION}

In many mammalian tissues, 5-, 12- and 15lipoxygenases exist, which convert arachidonic acid (5,8,11,14-icosatetraenoic acid) into hydroperoxyand hydroxy-icosatetraenoic acids, leukotrienes, hepoxilins and lipoxins. Icosanoids formed in the lipoxygenase pathways are of potential interest in regards to pancreatic islet function, in that nonselective inhibitors of lipoxygenases consistently attenuate stimulated insulin release in vitro (1-6).

In search for the identity of the putative insulin secretagogue, individual exogenous lipoxygenasepathway products have been administered to the pancreas or pancreatic islets. Among the hydroxyicosatetraenoic acids (HETEs), Yamamoto et al (7) found that 5-HETE stimulated basal insulin release; on the other hand, 12-HETE and 15-HETE inhibited glucose-induced insulin release. Metz et al (4) did not observe any effect of 12-HETE in neonatal rat islets; yet, Turk et al (1) reported that the attenuation of glucose-induced insulin release

Date received 20 September 1989

Date accepted 20 November 1989 evoked by a lipoxygenase inhibitor, icosatetraynoic acid (ETYA), was reversed partially by the addition of 12-HETE. Among the hydroperoxyicosatetraenoic acids (HPETEs), 12-HPETE and 15-HPETE stimulated insulin release in neonatal, but not in adult rat islets (8). Yamamoto et al (7) found that 15-HPETE inhibited glucose-induced insulin release. An epoxide formed in the 12-lipoxygenase pathway, 8-hydroxy-11, 12-epoxyicosatrienoic acid (hepoxilin $\mathrm{A}_{3}$ ) stimulated insulin release in the presence of $10 \mathrm{mM}$ glucose (9). We reported that 5,12 -dihydroxy-icosatetraenoic acid (leukotriene $\mathrm{B}_{4}, \mathrm{LTB}_{4}$ ), 5-hydroxy-6-S-glutathionylicosatetraenoic acid $\left(\mathrm{LTC}_{4}\right)$, and to a lesser extent 5-hydroxy-6-S-cysteinylglycinyl- icosateraenoic acid $\left(\mathrm{LTD}_{4}\right)$ and 5-hydroxy-6-S-cysteinyl- icosatetraenoic acid $\left(\mathrm{LTE}_{4}\right)$ stimulated insulin secretion from the isolated, perfused rat pancreas (10). Metz et al (4) found that an epoxy-derivative of $\mathrm{LTA}_{4}$ stimulated insulin release from neonatal rat islets.

Evidence has been sought for the biosynthesis of lipoxygenase-pathway products of arachidonate in pancreatic islets. A major icosanoid formed from arachidonate in islets is 12-HETE $(11,12)$. Pace-Asciak and Martin (9) rcportcd that rat islets produce 
hepoxilin $\mathrm{A}_{3}$. We provided preliminary evidence for the production of leukotrienes (13); although Turk et al $(12,14)$ failed to confirm this finding.

A potential interplay between the lipoxygenase pathway products and glucose in islets is suggested. First, an increase in ambient glucose concentration leads to increased production of these icosanoids in islets $(11,15)$. Second, the attenuation of insulin secretion with inhibitors of lipoxygenases has been demonstrated mostly with glucose-induced insulin release. The present study represents a unifying effort to establish which icosanoids formed in the three lipoxygenase pathways are secretagogues of insulin, and whether their actions are influenced by glucose in isolated rat islets. Our results indicate that $\mathrm{LTC}_{4}$ and hepoxilin $\mathrm{A}_{3}$ stimulate insulin release at a "basal" concentration of glucose, and, with the exception of $\mathrm{LTC}_{4}$, all lipoxygenase products tested inhibit glucose-induced insulin release.

\section{MATERIALS AND METHODS}

The supplier of collagenase (type V) and Ficoll was Sigma, St. Louis, MO, and that of 12-(R)-HETE 12(S)-HETE, 12-(S)-HPETE, LTB $_{4}$, LTC $_{4}$, hepoxilin $A_{3}$ as methyl ester, and 5, 6, 15-trihydroxyicosatetraenoic acid (lipoxin $B_{4}$ ) as methyl ester Cayman Chemical, Ann Arbor, MI. All icosanoids were shipped in dry ice in ethanol and were stored at $-70^{\circ} \mathrm{C}$.

On the day of each experiment, pancreatic islets were isolated from 250 to $350 \mathrm{~g}$ male Wistar rats, which had free access to food and water until the time of the procedure. The method of Gotoh et al (16) was used, in which the digestion of the pancreas proceeded without shaking, followed by a density gradient and manual sorting of the islets. Ten islets were transferred into each test tube, washed with Krebs-Ringer-Hepes buffer (KRH), and preincubated in $200 \mu \mathrm{KRH}$ containing $5.6 \mathrm{mM}$ glucose and $0.1 \%$ bovine serum albumin,

for $60 \mathrm{~min}$ at $37^{\circ} \mathrm{C}$ in an atmosphere of $95 \% 0_{2}$ and $5 \% \mathrm{CO}_{2}$. Upon completion of the preincubation, icosanoids or their vehicle ( $100 \%$ ethanol) appropriately diluted in a volume of $400 / \mu \mathrm{l}$ and $400 \mu \mathrm{l}$ of buffer containing glucose to yield a final glucose concentration of either $5.6 \mathrm{mM}$ or $23 \mathrm{mM}$ were added to each test tube. The final concentrations of the icosanoids were: 12-(R)-HETE or 12-(S)-HETE $10^{-6} \mathrm{M} ; 12$-(S)-HPETE $10^{-6} \mathrm{M} ;$ LTB $_{4} 10$ ${ }^{6} \mathrm{M} ; \mathrm{LTC}_{4} 6 \times 10^{-7} \mathrm{M}$; hepoxilin $\mathrm{A}_{3} 10^{-6} \mathrm{M}$; and lipoxin $\mathrm{B}_{4} \mathrm{M}$. The 1.0-ml samples were incubated for $30 \mathrm{~min}$ at $37^{\circ} \mathrm{C}$. Thereafter, the tubes were chilled rapidly and centrifuged at $800 \mathrm{~g}$ for $3 \mathrm{~min}$ at $4^{\circ} \mathrm{C}$. The supernatants were removed and stored at $-20^{\circ} \mathrm{C}$ for subsequent measurement of the levels of insulin.

The levels of insulin in the incubation media were measured by a 'double-antibody' radioimmunoassay, in which a rat insulin antiserum and rat insulin standards were employed (17). The interassay variability was $6-9 \%$.

At least three experiments of each kind were carried out, each time using a different batch of islets. Each experimental condition was run in triplicate. On each sample, the radioimmunoassay was carried out in duplicate, and the mean result of the replicates was computed. To accommodate the variation in insulin-secretory capacity among different batches of islets, the data for each experiment were normalized by setting the result obtained with $5.6 \mathrm{mM}$ glucose alone to $100 \%$. The means from all experiments of the same kind were used to obtain the mean \pm SEM for that test condition. The statistical significance of the differences among means was determined by analysis of variance using Dunnett's tetesting.

Table Immunoreactive insulin release from isolated rat islets in response to icosanoids formed in various lipoxygenase pathways, in the presence of low and high concentrations of glucose

\begin{tabular}{|c|c|c|c|c|}
\hline \multirow[t]{2}{*}{ Icosanoid } & \multirow[t]{2}{*}{$(\mathrm{N})$} & \multicolumn{3}{|c|}{$\begin{array}{l}\text { Insulin Release (Mean } \pm \text { SEM Percent)* } \\
\text { With } 5.6 \mathrm{mM} \text { Glucose } \\
\text { With } 23 \mathrm{mM} \text { Glucose }\end{array}$} \\
\hline & & Icosanoid & No Icosanoid & Icosanoid \\
\hline $\begin{array}{l}\text { LTB }_{4} ; 10^{6} \mathrm{M} \\
\text { LTC }_{4} ; 6 \times 10^{7} \mathrm{M} \\
\text { 12-(S)-HPETE; } 10^{-6} \mathrm{M} \\
\text { 12-(R)-HETE;10-6 M } \\
\text { 12-(S)-HETE; } 10^{-6} \mathrm{M} \\
\text { Hepoxilin } \mathrm{A}_{3} ; 10^{6} \mathrm{M} \\
\text { Lipoxin } \mathrm{B}_{4} ; 10^{-6} \mathrm{M}\end{array}$ & $\begin{array}{l}(3) \\
(3) \\
(3) \\
(4) \\
(4) \\
(3) \\
(3)\end{array}$ & $\begin{array}{l}136 \pm 12 \\
304 \pm 32 \dagger \\
56 \pm 6 \dagger \\
111 \pm 19 \\
104.5 \pm 6.6 \\
234 \pm 39 \dagger \\
106.4 \pm 12\end{array}$ & $\begin{array}{l}250 \pm 51 \dagger \\
250 \pm 51 \dagger \\
139 \pm 11 \dagger \\
190 \pm 10 \dagger \\
190 \pm 10 \dagger \\
320 \pm 33 \dagger \\
320 \pm 33 \dagger\end{array}$ & $\begin{aligned} 130 & \pm 19 \dagger \\
162 & \pm 15 \\
50 & \pm 5 \dagger \\
91 & \pm 14 \dagger \\
112 & \pm 18 \dagger \\
145 & \pm 28 \dagger \\
101 & \pm 27 \dagger\end{aligned}$ \\
\hline
\end{tabular}

Footnotes:

The final concentration of each icosanoid is given in parentheses next to its name.

For each experimental condition, the number of observations is given in parentheses.

$\left[{ }^{*}\right]$ The data for each experiment were normalized by setting the result obtained with $5.6 \mathrm{mM}$ glucose alone to $100 \%$.

It $\mathrm{p}<0.05$ vs with $5.6 \mathrm{mM}$ glucose alone.

I $\dagger \mathrm{p}<0.05$ vs with $23 \mathrm{mM}$ glucose alone. 


\section{RESULTS}

When the data from all experiments were compiled, over the 30-min incubation period, in the absence of any icosanoids, the secretion of insulin was 229 $\pm 40 \mu \mathrm{U} /$ islet $/ \mathrm{h}$ with $5.6 \mathrm{mM}$ glucose and $410 \pm 55$ $\mu \mathrm{U} /$ islet/h with $23 \mathrm{mM}$ glucose $(\mathrm{N}=13 ; \mathrm{p}<0.01)$. The data on the effects of individual icosanoids at the two concentrations of glucose are given in the Table.

The effects of two 5-lipoxygenase pathway products were tested. Under basal conditions (5.6 mM glucose), $\mathrm{LTC}_{4}$ stimulated the release of insulin in the presence of $5.6 \mathrm{mM}$ glucose; $\mathrm{LTB}_{4}$ had a mild stimulatory effect which did not attain statistical significance. The stimulatory effect of $\mathrm{LTC}_{4}$ exceeded that of $\mathrm{LTB}_{4}(304 \%$ vs $136 \%$, p $<0.05)$. LTC $_{4}$ did not modify the release in response to $23 \mathrm{mM}$ glucose; on the other hand, $\mathrm{LTB}_{4}$ inhibited glucose-induced insulin release.

Four 12-lipoxygenase pathway products were selected. 12-(S)-HPETE inhibited insulin release in the presence of $5.6 \mathrm{mM}$ as well as $23 \mathrm{mM}$ glucose. Neither 12-(R)-HETE nor 12-(S)-HETE had any effect on basal insulin release. In the presence of $23 \mathrm{mM}$ glucose, 12-(R)-HETE as well as 12-(S)HETE inhibited glucose-induced insulin release. Hepoxilin $A_{3}$ stimulated insulin secretion in the presence of $5.6 \mathrm{mM}$ glucose, but inhibited insulin release evoked by $23 \mathrm{mM}$ glucose.

Both 5-lipoxygenase and 15-lipoxygenase are involved in the biosynthesis of lipoxin. $\mathrm{B}_{4}$ Lipoxin had no effect on basal insulin secretion. It inhibited insulin release stimulated by $23 \mathrm{mM}$ glucose.

Statistical comparison of the insulin-secretory responses to individual lipoxygenase-pathway products in the presence of $5.6 \mathrm{mM}$ glucose $v s$ in the presence of $23 \mathrm{mM}$ glucose did not reveal any significant differences.

\section{DISCUSSION}

Our results indicate that icosanoids formed in the lipoxygenase pathways affect insulin secretion in a complex manner.

Since $5.6 \mathrm{mM}$ is slightly below the blood level of glucose which prevails in the fasting rat, and since the islets had been exposed to this concentration during the 60 - $\mathrm{min}$ preincubation followed by the 30 min incubation periods, we assumed that the insulin secreted with $5.6 \mathrm{mM}$ glucose represented the 'basal' secretion, and interpreted the response to an icosanoid at this concentration of glucose as an effect of that icosanoid on its own.

Among the seven icosanoids representing the products formed in the three lipoxygenase pathways, the 5-lipoxygenase product $\mathrm{LTC}_{4}$ and the 12-lipoxygenase product hepoxilin $\mathrm{A}_{3}$ stimulated the basal secretion of insulin. In this regard, $\mathrm{LTC}_{4}$ was by far the more potent one, despite the fact that it was employed at a concentration lower than all other icosanoids. We reported previously that, in the perfused rat pancreas, in the presence of $5.6 \mathrm{mM}$ glucose, $\mathrm{LTB}_{4}$ as well as $\mathrm{LTC}_{4}$ stimulate insulin secretion at similar potencies in a dose-related manner in the concentration range of $10^{-11} \mathrm{M}$ to $10^{-7}$ $M(10)$. In the present study, the mild stimulatory effect of $\mathrm{LTB}_{4}$, at a concentration higher than those employed previously, did not attain statistical significance. In addition to the obvious differences in the experimental conditions, the possibility of tachyphylaxis may be considered. Our observation of an agonist action of hepoxilin $\mathrm{A}_{3}$ in the presence of $5.6 \mathrm{mM}$ glucose is an extension of that of PaceAsciak and Martin (9) that hepoxilin stimulated insulin release at $10 \mathrm{mM}$ glucose.

Thus, to this date, among the lipoxygenasepathway products, $\mathrm{LTB}_{4}$ and peptidyl LTs $(10$, and present study), 5-HETE (7), and hepoxilin $A_{3}$ (9) have been shown to stimulate basal insulin secretion on their own.

In our study, 12-(S)-HPETE inhibited the basal release of insulin. Metz et al (4) reported that 12HPETE stimulated insulin release in neonatal rat islets, but had no effect in adult islets. The reasons for the age difference in the insulin-secretory responses observed by Metz, and the contrasting inhibitory response which we observed remain to be clarified; differences in experimental conditions, or the fact that we used selectively the (S) enantiomer rather than an unspecified preparation of 12HPETE may have affected the outcome. 12-HPETE is chemically unstable; conversion of 12 HPETE to another product in islets may be considered as being responsible for the inhibitory effect. 12-HPETE is the immediate precursor of 12-HETE; reportedly, 12-HPETE can be converted also to hepoxilin in islets (9). Our results with exogenous 12-HETEs or hepoxilin do not lend support to the possibility that these compounds mediated the inhibitory effect of 12-HPETE, in that 12-HETE had no effect on, and hepoxilin stimulated basal insulin release. Nevertheless, some other compound formed in islets from 12-HPETE may have been responsible for the inhibition.

Neither the (R) nor the (S) enantiomer of 12 HETE stimulated the basal secretion of insulin. In previous studies, 12-HETE preparations of unknown type had been used. We had considered that stereospecificity may be a determinant in any secretagogue action; for example, the (R) enantiomer of 12-HETE may interact with $\mathrm{LTB}_{4}$ receptors (18). Still, the employment of the individual enantiomers of 12-HETE failed to uncover an agonist action which previously may have been overlooked. 
The effect of lipoxins on the secretion process previously had not been tested. Lipoxin $\mathrm{B}_{4}$ failed to affect basal insulin secretion. In the case of lipoxin, as well as in the case of the two enantiomers of 12 HETE, one may consider that they were inhibitory in the basal state, but that the model employed was not sufficiently sensitive to demonstrate the inhibition. This is unlikely, since the inhibitory action of 12-(S)-HPETE could be revealed.

The striking novel finding in our study is that, with the exception of $\mathrm{LTC}_{4}$, all lipoxygenase pathway products employed exerted an inhibitory action on insulin release, when insulin release had been augmented in the presence of $23 \mathrm{mM}$ glucose. In most cases, these icosanoids suppressed insulin release toward or to, but not below the levels observed in the presence of $5.6 \mathrm{mM}$ glucose alone. Hence, we believe that the lipoxygenase products interfered with the stimulatory signal for insulin secretion elicited by glucose, irrespective of whether, on their own, they may have a stimulatory or inhibitory effect, or no effect on insulin release. An extrapolation of our findings with exogenous lipoxygenase-pathway products to their endogenous counterparts would assign to the latter a negative rather than a positive modulatory role in glucoseinduced insulin release. Since glucose promotes the biosynthesis of icosanoids in islets, including the lipoxygenase pathway products, the intriguing possibility emerges that, by inducing the increased availability of these compounds, glucose engages a restraining system on insulin release.

$\mathrm{LTC}_{4}$ stimulated basal insulin release and had no effect on glucose-induced insulin release. In the case of hepoxilin, the stimulation of basal, but inhibition of glucose-induced insulin release are seemingly paradoxical. These icosanoids may have two or more mechanisms of action in the islet B-cells. One mechanism may provide for a tonic-stimulatory influence, while another may interfere selectively with the signal for insulin release generated by glucose. The latter mechanism may have mitigated the strong agonist action $\mathrm{LTC}_{4}$ only partially, while it may have overwhelmed the action of the weaker agonist hepoxilin. In this regard, the lipoxygenase products are not unique. The promotion of the basal secretion of insulin by prostaglandins has been observed repeatedly (19-21); yet, others reported that the same icosanoids inhibited glucose-induced insulin release $(20,22)$.

The results of our study, as well as those of previous work by others, do not provide an explanation for the consistent observation that nonselective inhibitors of lipoxygenases inhibit stimulated insulin release. All drugs are likely to have actions in addition to the main action attributed to them; hence, these compounds may be inhibiting insulin release by mechanisms unrelated to the inhibition of lipoxygenases. The fact that chemically unrelated lipoxygenase inhibitors have the same effect weakens this possibility. Another possibility is that the inhibition of lipoxygenases may lead to increased production in alternate pathways of certain arachidonate metabolites that could be inhibitors of stimulated insulin release. A third possibility is that other icosanoids formed in islets in the lipoxygenase pathways, the identity of which is obscure at this time, may have tonic-stimulatory properties without interfering with the glucose signal, the formation of which is inhibited by the lipoxygenase inhibitors.

We conclude that, among the lipoxygenase products tested, only leukotrienes and hepoxilin are candidates for a tonic-stimulatory influence on basal insulin release. Our observation that lipoxygenase products inhibited glucose-induced insulin release, together with the existing evidence that glucose augments icosanoid formation in pancreatic islets, suggest the existence in islets of a negative-feedback system involving these icosanoids.

\section{Acknowledgements}

The study was supported in part by the United States Public Health Service grants R01 DK21192, T32 DK07245, F32 DK08282 and P60 DK20572 from the National Institute of Diabetes, and Digestive and Kidney Diseases.

\section{References}

1. Turk J, Colca J R, McDaniel M L. Arachidonic acid metabolism in isolated pancreatic islets. III Effects of exogenous lipoxygenase products and inhibitors on insulin secretion. Biochim Biophys Acta 834: 23-36, 1985

2. Turk J, Wolf B A, McDaniel M L. The role of phospholipid-derived mediators including arachidonic acid, its metabolites, and inositoltriphosphate and of intracellular $\mathrm{Ca}^{2+}$ in glucose-induced insulin secretion by pancreatic islets. Progr Lipid Res 26: 125-181, 1987.

3. Morgan R, Pek S B. Role of arachidonate lipoxygenase and cyclooxygenase products in insulin and glucagon secretion from rat pancreatic islets. Metabolism 33: 928-935, 1984.

4. Metz S, Van Rollins M, Strife R, Fujimoto W, Robertson R P. Lipoxygenase pathway in islet endocrine cells: Oxidative metabolism of arachidonic acid promotes insulin release. J Clin Invest 71: 1191-1205, 1983.

5. Walsh M F, Pek S B. Possible role of endogenous arachidonic acid metabolites in stimulated release of insulin and glucagon from the isolated rat pancreas. Diabetes 33: 929-936, 1984.

6. Walsh M F, Pek S B. Effects of lipoxygenase and cyclooxygenase inhibitors on glucose-stimulated insulin secretion from the isolated perfused rat pancreas. Life Sci 34: 1699-1706, 1984.

7. Yamamoto S, Ishii M, Nakadate T, Nakaki T, Kato R. Modulation of insulin secretion by lipoxygenase products of arachidonic acid. J Biol Chem 258: 12149-12152, 1983.

8. Metz S A, Murphy R C, Fujimoto W Y 1984 Effects on glucose-induced insulin secretion of lipoxygenase-derived metabolites of arachidonic acid. Diabetes 33: 119-124. 
9. Pace-Asciak C R, Martin J M. Hepoxilin, a new family of insulin secretagogues formed by intact rat pancreatic islets. Prostaglandins Leukotrienes Med 16: 173-180, 1984.

10. Pek S B , Walsh M F. Leukotrienes stimulate insulin release from the rat pancreas. Proc Natl Acad Sci USA 81: 2199-2202, 1984.

11. Metz S. Glucose increases the synthesis of lipoxygenase-mediated metabolites of arachidonic acid in intact rat islets. Proc Natl Acad Sci USA 82: 198-202, 1985 .

12. Turk J, Colca J R, Kotagal N, McDaniel M L. 1984. Arachidonic acid metabolism in isolated pancreatic islets. 1. Identification and quantitation of lipoxygenase and cyclooxygenase products. Biochim Biophys Acta 794: 110-124.

13. Pek S B, Walsh M F. Effects of icosanoids on in vitro secretion of islet hormones. Excerpta Medica Intl Cong Ser 700: 198-202, 1986

14. Turk J, Wolf B A, Comens P G, Colca J, Jakschik B, McDaniel M L. Arachidonic acid metabolism in isolated pancreatic islets. IV. Negative ion-mass spectrometric quantitation of monooxygenase producty synthesis by liver and islets. Biochim Biophys Acta 835: 1-17, 1985.

15. Turk J, Colca J R, Kotagal N, McDaniel M L. Arachidonic acid metabolism in isolated pancreatic islets. II. The effects of glucose and of inhibitors of arachidonate metabolism on insulin secretion and metabolic synthesis. Biochim Biophys Acta 794: $125-136.1984$

16. Gotoh M, Maki T, Kiyoizumi T, Satomi S, Monaco A P. An improved method for isolation of mouse pancreatic islets. Transplantation 40: 437-438, 1985.

17. Pek S. Glucagon and insulin. pp 122-140 in Nuclear Medicine: Endocrinology. (Rothfeld B ed) Lippincott. Philadelphia, 1978.

18. Evans J F, Leblanc Y, Fítzsimmons B J, Charleson $S$, Nathaniel $D$, Leveille $C$. Actuvation of leukocyte movement and displacement of ${ }^{3} \mathrm{H}-\mathrm{LTB}_{4}$ from leukocyte membrane preparations by $12(R)$ - and 12(S)-hydroxyeicosatetraenoic acid. Biochim Biophys Acta 917: 406-410, 1987

19. Johnson D G, Fujimoto W Y, Williams R $\mathbf{H}$ Enhanced release of insulin by prostaglandins in isolated pancreatic islets. Diabetes 22: 658-663. 1973.

20. Burr I, Sharp R. Effects of prostaglandin $E_{1}$ and epinephrine on the dynamics of insulin release in vitro. Endocrinulogy 94: 835-839, 1974.

21. Pek S, Tai T-Y, Elster A. Stimulatory effects of prostaglandins $E_{1}, E_{2}$ and $F_{7}$ alpha on glucagon and insulin release in vitro. Diabetes 27: 801-809, 1978.

22. Robertson R P. Arachidonic acid metabolite regulation of insulin secretion. Diabetes Metab Rev 2: 261-296, 1986. 